\title{
La surveillance individuelle de l'exposition interne aux oxydes d'uranium* dans deux usines de fabrication du combustible
}

\author{
F. BOURDEIX ${ }^{\star \star}, \mathrm{Ph}$. BERARD ${ }^{\star \star \star}$, J. ACHIARY $\star \star$
}

(Manuscrit reçu le 28 octobre 1988)

\begin{abstract}
RÉSUME
La surveillance individuelle du personnel exposé à l'inhalation d'oxydes d'uranium au cours de la fabrication du combustible pour réacteurs à eau sous pression (R E P) associe la spectrométrie gamma du thorax, la mesure de l'excrétion fécale et urinaire de l'uranium. L'analyse des résultats montre que l'exposition interne résulte d'incidents répétitifs qu'on peut mettre en évidence par des pics précoces d'élimination urinaire et surtout fécale, qu'il existe souvent un décalage par rapport aux résultats d'ambiance donnés par les appareils de prélèvement d'air (APA), et que les variations mesurées de la charge thoracique en uranium sont relativement rapides (moyenne des périodes apparentes $165 \mathrm{j}$ ).

L'évaluation de l'équivalent de dose effectif dû à l'inhalation demande les précisions suivantes: granulométrie de l'aérosol aux postes de travail, caractéristiques physico-chimiques du produit en vue d'apprécier sa solubilité biologique réelle, mesure de la fraction inhalable à l'aide d'un appareil de prélèvement individuel portatif.
\end{abstract}

\section{ABSTRACT}

Individual monitoring of personal exposure to inhalation of uranium oxides throughout the manufacture of fuel for pressurized water reactor (PWR) includes lung gamma-spectrometry, fecal analysis and urine analysis. Examination of the results shows the following: internal exposure is the consequence of repeated intake incidents as revealed by early peaks of urinary and particularly fecal elimination; a shift is often observed with the results of aerosol concentration measured through air collectors; the mesured variations of uranium lung incorporations are relatively fast (apparent mean period $165 \mathrm{~d}$ ). Correct evaluation of the effective dose equivalent from inhalation requires further information concerning the aerosol size distribution at work stations, the physico-chemical characteristics of the product leading to an estimate of its actual biological solubility, and the measurement of the fraction of aerosol liable to intake with an individual portable collector.

\footnotetext{
"Communication présentée aux journées "Uranium", Montpellier, 10-13 octobre 1988.

** Service médical du travail, Société franco-belge de fabrication de combustibles FBFC. Les Bérauds, 26104 Romans-sur-Isère Cedex et BP 3, 26701 Pierrelatte Cedex.

*** Commissariat à l'énergie atomique, IPSN, Département de protection sanitaire, Service d'hygiène industrielle, BP 38, 26701 Pierrelatte Cedex.
} 


\section{INTRODUCTION}

\subsection{Les produits}

L'exposition des travailleurs dans les usines de fabrication du combustible est due principalement à I' $\mathrm{O}_{2}$ (dioxyde) et à l' $\mathrm{U}_{3} \mathrm{O}_{8}$ (sesquiosyde), tous deux classés non transférables (classe $\mathrm{Y}$ ) par la Commission internationale de protection radiologique (CIPR). Le risque à prendre en compte est donc le risque radiologique seul, la porte d'entrée étant l'inhalation.

\subsection{Métabolisme}

Le modèle pulmonaire donné par la publication $n^{\circ} 30$ de la CIPR montre que le dépôt dépend de la granulométrie de l'aérosol inhalé (précisément du diamètre aérodynamique médian en activité DAMA). L'épuration des particules inhalées se fait par deux voies:

- Le tube digestif après remontée le long des voies respiratoires et passage du carrefour aéro-digestif; c'est la voie principale; l'excrétion fécale représente $1 / 1000$ de l'inhalation au jour 10, après une excrétion précoce importante liée à la fraction haute du dépôt, de grosse granulométrie [6];

- le rein, après passage lent de l'uranium dans la circulation systémique; au jour 10, l'excrétion urinaire représente $1 / 10000$ de l'inhalation.

Le modèle est donné par la CIPR pour un DAMA de $1 \mu \mathrm{m}$ et une période biologique de $500 \mathrm{j}$; la réalité industrielle pourra être différente, d'où l'intérêt des mesures physiques (granulométrie, enrichissement, densité, surface spécifique, spectre de diffraction aux rayons $\mathrm{X}$ ) et de l'étude de la solubilité in vitro.

\subsection{Circonstances de l'exposition}

La contamination provient des multiples ruptures de confinement qui peuvent exister:

- soit en marche normale: transferts de poudre, récupération des pastilles en sortie de presse, prélèvements pour contrôle, nettoyage pour changement d'enrichissement;

- soit en fonctionnement incidentel: fuite sur une installation, faute de procédure d'un opérateur entraînant la mise en suspension de poudre.

\section{MÉTHOdE DE SURVEILLANCE}

\subsection{Collaboration avec le Service de protection contre les rayonnements (SPR)}

La mise en place d'un protocole de surveillance de l'exposition interne nécessite la connaissance de la contamination aérienne et des circonstances dans lesquelles elle s'est produite: nature du radiocontaminant (cf. supra), niveau de la contamination, sa localisation dans le temps (aiguë ou chronique) et dans l'espace (répartition dans les locaux de travail). 
La surveillance des installations et de l'ambiance de travail est réalisée par le SPR (notamment grâce aux appareils de prélèvements d'air APA); elle permet de connaître la teneur moyenne de l'air en uranium sur $8 \mathrm{~h}$ ou $24 \mathrm{~h}$ de travail à différents postes. Cette valeur, donnée par l'APA, est souvent la résultante de plusieurs pics de contamination ponctuels, mais elle permet, dans tous les cas, de saisir une dérive due à un incident significatif.

L'analyse permanente de la contamination atmosphérique par le SPR donne donc au médecin du travail des informations indispensables pour la conduite de la surveillance individuelle.

\subsection{Stratégie de prélèvement}

Elle découle de ce qui vient d'être dit:

- en cas d'incident de contamination: recherche de l'excrétion urinaire précoce: urine de $24 \mathrm{~h}$ ou échantillonnage de $250 \mathrm{ml}$ répété le $1^{\text {er }}$ et le $2^{\text {ème }} \mathrm{j}$ (taux d'uranium rapporté à la créatine) et uranium fécal $(72 \mathrm{~h}$ );

- la surveillance systématique est modulée en fonction de l'appréciation du risque (reposant sur l'information donnée par le SPR et aussi sur la synthèse de la surveillance individuelle antérieure); elle associe la spectrométrie au niveau du thorax (de 1 fois tous les deux ans à 2 fois par an) et la recherche de l'excrétion fécale (en alternance au même rythme). L'uranium fécal est recherché après au moins 15 jours d'arrêt de l'exposition.

Enfin, des examens sont pratiqués de façon ponctuelle pour contrôle ou sondage, qu'il s'agisse d'évaluer le risque à un nouveau poste ou de vérifier l'absence de dérive dans un atelier où l'exposition est en principe négligeable (exemple: le laboratoire d'analyse).

Outre les examens précédemment cités, on peut utiliser la recherche de l'uranium recueilli dans un mouchoir en papier en fin de poste. C'est un examen d'orientation simple et qui peut être très utile.

\subsection{Valeurs de référence [2] ${ }^{*}$}

En routine, on utilise des limites dérivées:

- pour la charge thoracique mesurée en spectrométrie gamma:

$-7,5 \mathrm{nCi}(280 \mathrm{~Bq})=$ niveau d'investigation dérivé (nid);

- $18 \mathrm{nCi}(670 \mathrm{~Bq})=$ niveau d'action (sachant que la charge thoracique à l'équilibre correspondant à l'inhalation permanente de la LDCA ou limite de concentration atmosphérique est de $25 \mathrm{nCi}$, soit $925 \mathrm{~Bq}$ );

\section{- pour l'excrétion fécale:}

- en surveillance systématique, si le recueil de l'échantillon est correct (selles de $72 \mathrm{~h}$ après $15 \mathrm{j}$ au moins d'interruption du travail), on considère

* Le groupe de travail GT $8 / 1$ rassemble médecins. biologistes et ingénieurs concernés par la surveillance de l'exposition interne aux composés d'uranium. 
que l'excrétion journalière représente le $1 / 1000^{\text {ème }}$ de la charge thoracique;

Après incident, une excrétion des premières $24 \mathrm{~h}$ de $350 \mathrm{pCi}(13 \mathrm{~Bq})$ correspond à la charge pulmonaire de $7,5 \mathrm{nCi}$.

- pour l'excrétion urinaire, il faut considérer que c'est un examen d'orientation et que toute valeur mesurable, dépassant le seuil de détection en méthode usuelle $(10 \mathrm{pCi} / 1$ soit $0,4 \mathrm{~Bq} / 1)$ correspond à 1 nid et nécessite une investigation complémentaire.

\section{RÉSULTATS}

Nous présentons ici trois exemples caractéristiques pour montrer la progression réalisée depuis 5 ans dans la connaissance des conditions réelles d'exposition, l'expérience acquise et le travail réalisé par le GT 8/1 permettant une optimisation progressive de la surveillance.

3.1. Exemple $n^{\circ} 1$ (fig. 1 et 2 )

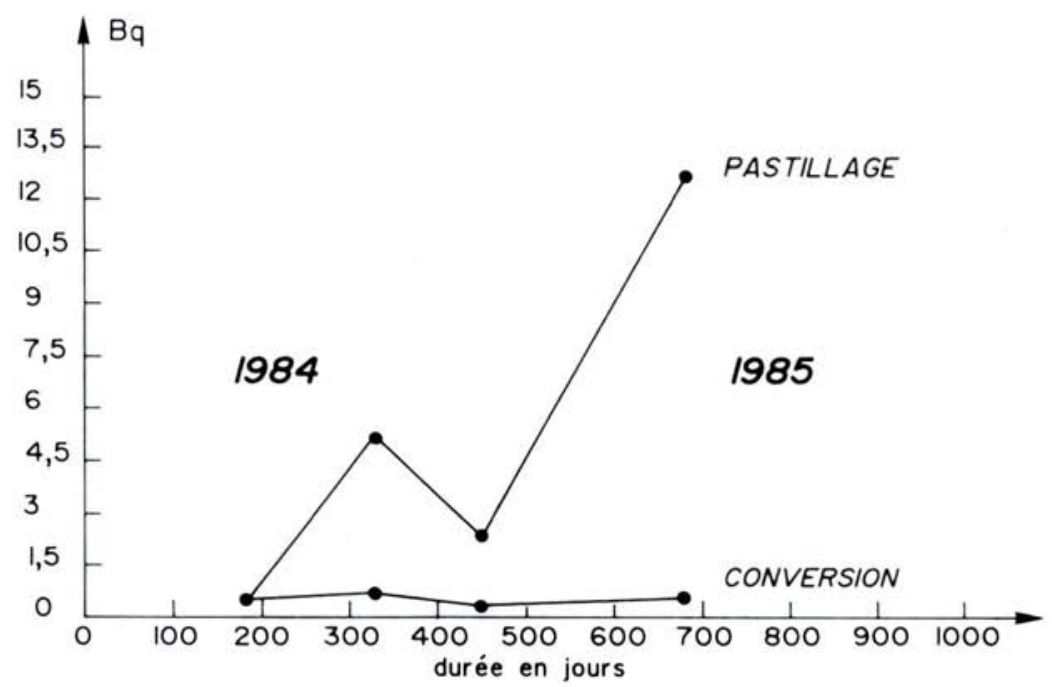

Fig. 1. - Evolution des moyennes des examens fécaux (72 h) sur deux années $\mathrm{UO}_{2}$ : uranium enrichi $3,25 \%$

II s'agit de mesures de rétention pulmonaire (2 fois par an), d'excrétion fécale ( 2 fois par an) et urinaire ( 4 fois par an) effectuées en 1984-1985 sur 10 ouvriers appartenant à 2 ateliers différents (pastillage et conversion). Les figures 1 et 2 prennent en compte les moyennes des données pour les ouvriers d'un même atelier. 


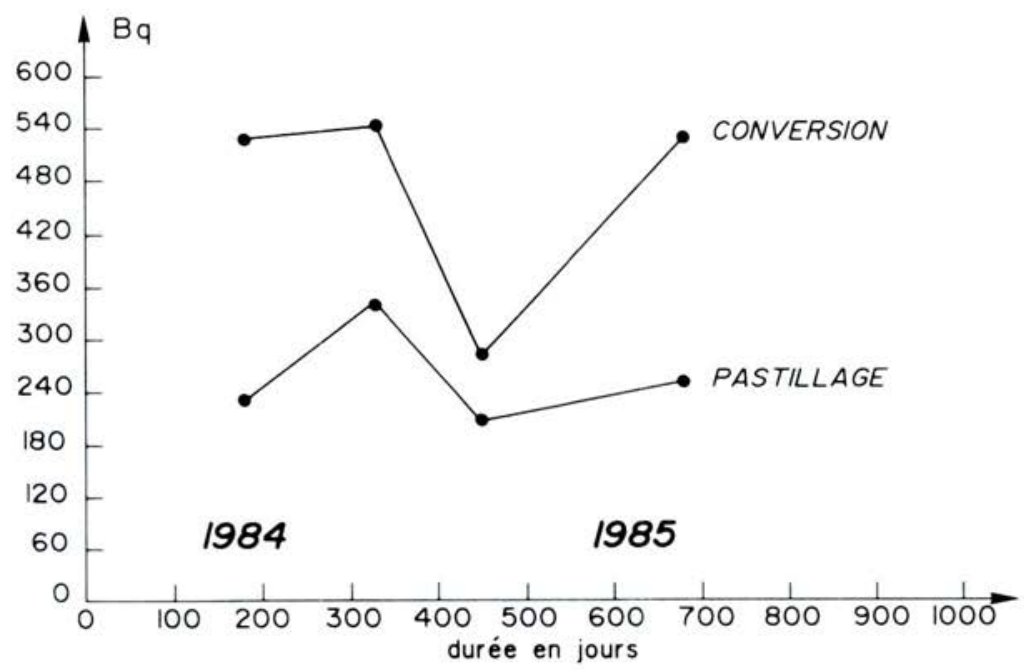

Fig. 2. - Evolution des moyennes des examens anthropogammamétriques sur deux années.

$\mathrm{UO}_{2}$ : uranium enrichi $3,25 \%$.

Les recherches d'uranium fécal ont èté réalisées après des arrêts d'exposition de durée variable (de 1 à $21 \mathrm{j}$ ); en particulier, le dernier résultat de l'atelier pastillage reflète l'importance de l'élimination fécale précoce (échantillon recueilli en fin de semaine sans arrêt de travail préalable).

Les résultats de spectrométrie gamma sont, sur cet échantillonnage réduit de 1984-1985, plus élévés qu'on pourrait l'attendre en fonction des chiffres donnés par les APA: à l'atelier de conversion notamment, la moyenne est de $12,7 \mathrm{nCi}(470 \mathrm{~Bq})$ pour une concentration dans l'air donnée par les APA de 0,2 de la CMA travailleur $\left(120 \mathrm{pCi} / \mathrm{m}^{3}\right.$ soit $4,5 \mathrm{~Bq} / \mathrm{m}^{3}$ pour $40 \mathrm{~h}$ de travail/5 j)

II est important de noter qu'en 3 ans l'effort de prévention a permis de faire baisser cette valeur d'un facteur 2: la moyenne des valeurs de rétention pulmonaire à l'atelier de conversion est, pour l'année 1988, inférieure à $6 \mathrm{nCi}(220 \mathrm{~Bq})$. Les variations de six mois en six mois de la charge pulmonaire paraissent plus importantes que ne l'autoriserait la période de $500 \mathrm{j}$ : la moyenne des périodes apparentes est de $165 \mathrm{j}$.

II existe une différence significative entre les résultats du pastillage et ceux de la conversion (en particulier la rétention pulmonaire moyenne au pastillage est de $7 \mathrm{nCi}$ soit $260 \mathrm{~Bq}$ ); les mesures de granulométrie réalisées par la suite à différents postes des deux ateliers ont permis de compléter l'analyse en montrant un DAMA de $3 \mu \mathrm{m}$ pour la conversion et de $6 \mu \mathrm{m}$ pour le pastillage, avec une bonne corrélation entre ce dernier paramètre et la rétention pulmonaire. 
Le rapport excrétion fécale/charge pulmonaire (lorsque l'arrêt de travail est superieur à $15 \mathrm{j}$ ) est d'environ 1/1000.

L'uranium urinaire n'est mesurable $(>7 \mu \mathrm{g} / \mathrm{l})$ que dans $18 \%$ des cas, ce qui confirme ses limites en surveillance systématique.

3.2. Exemple $n^{\circ} 2$ (fig. 3)

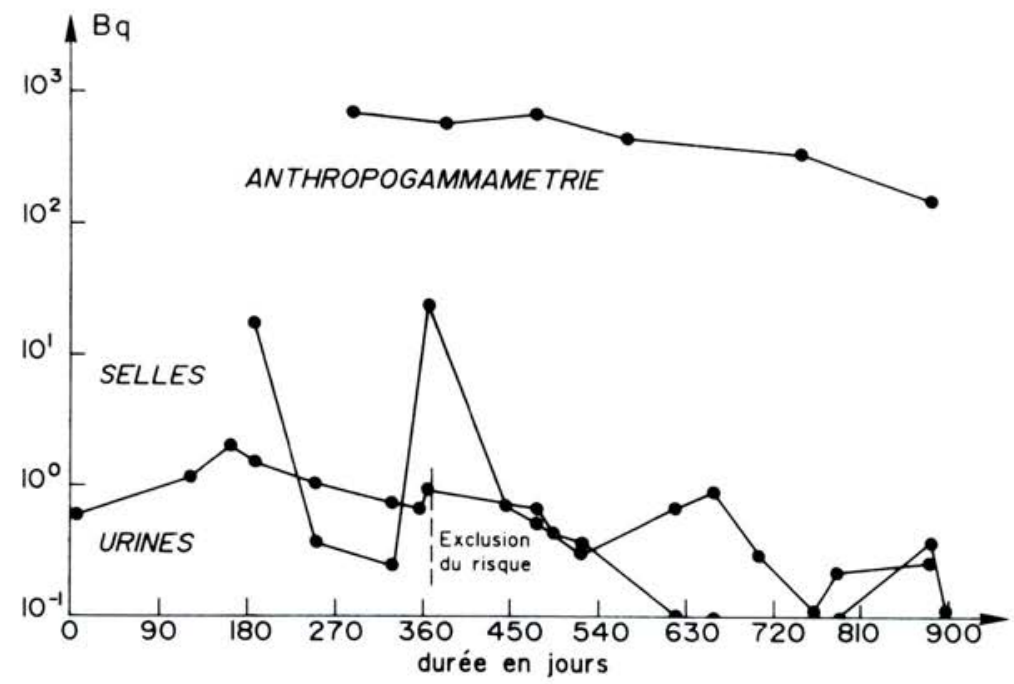

Fig. 3. - Évolution des résultats radiotoxicologiques (urines, selles, spectro) avant et après changement de poste.

II s'agit d'un ouvrier changé de postè après avoir atteint $17 \mathrm{nCi}$ $(670 \mathrm{~Bq})$ en spectrométrie du thorax. L'évolution des mesures thoraciques étalées sur huit mois montre une période de $165 \mathrm{j}$ et un rapport excrétion fécale/charge pulmonaire de $1 / 1000^{\mathrm{eme}}$. Les mesures réalisées avant exclusion montrent fort bien les pics d'excrétion précoce fécale, et, à un moindre degré, urinaire.

La quantification de la dose engagée aux poumons pour cet ouvrier donne 0,09 Sv soit 9 rem dans l'année (soit 1/5 de la limite de dose annuelle) avec comme base de calcul:

- l'évolution des résultats des examens anthropogammamétriques et une période de rétention de $165 \mathrm{j}$,

- le modèle pulmonaire de la CIPR 30 ,

- une exposition à des aérosols d'un composé Y d'un DAMA de $1 \mu \mathrm{m}$,

- un facteur de qualité pour les alpha de 20. 
3.3. Exemple $n^{\circ} 3$ (fig. 4)

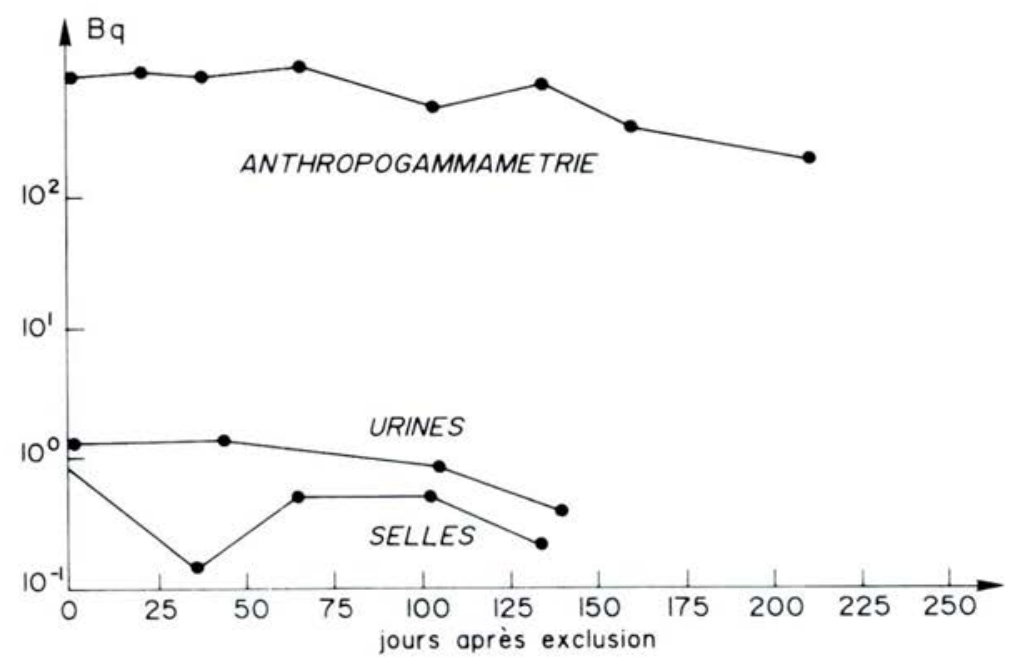

Fig. 4. - Evolution des examens après exclusion. Aérosols de classe $\mathrm{Y}$ : $\mathrm{UO}_{2}, \mathrm{U}_{3} \mathrm{O}_{8}$, pastillage.

A l'occasion d'un examen systématique, l'anthropogammamétrie d'un agent a révélé une charge pulmonaire légèrement supérieure au niveau d'action (18 $\mathrm{nCi}$ soit $670 \mathrm{~Bq}$ ). Après confirmation de ce résultat (contrôle), l'exclusion du risque a été décidée et une surveillance mensuelle de l'uranium fécal, urinaire et de la charge pulmonaire instituée afin d'en suivre l'évolution. La figure 4 montre qu'au bout de 6 mois, l'ensemble des résultats témoigne d'une rétention faible $(150 \mathrm{~Bq})$. L'agent a pu reprendre son poste. On remarque:

- la période de décroissance calculée est de $140 \mathrm{j}$,

- le rapport excrétion fécale/charge pulmonaire est de 1/1000,

- l'excrétion urinaire est plus importante que l'excrétion fécale.

La quantification de la dose engagée aux poumons pour cet agent donne 0,08 Sv soit 8 rem dans l'année (soit $1 / 5$ de la limite de dose annuelle) avec comme base de calcul:

- l'évolution des résultats des examens anthropogammamétriques et une période de rétention de $140 \mathrm{j}$,

- le modèle de la CIPR 30,

- une exposition à des aérosols d'un composé $\mathrm{Y}$ d'un DAMA de $1 \mu \mathrm{m}$,

- un facteur de qualité pour les alpha de 20. 


\section{CONCLUSIONS}

La surveillance individuelle des travailleurs exposés aux oxydes d'uranium pose plusieurs problèmes:

- la détermination des populations à surveiller de manière permanente et de celles pour lesquelles on ne fera des mesures que ponctuellement (sur incident ou pour des travaux particuliers ou pour sondage),

- la mise au point d'un protocole de surveillance fiable, permettant d'évaluer la dose interne et qui soit compatible avec l'organisation du travail.

L'expérience acquise à $\mathrm{FBFC}$ et au $\mathrm{SHI}$ montre que la réponse à ces questions nécessite une bonne collaboration du médecin du travail avec le responsable de la radioprotection et son équipe, d'une part, le laboratoire, les biologistes et spécialistes de radiotoxicologie, d'autre part.

En effet, pour l'évaluation de la dose interne, il est nécessaire de connaître:

- les résultats des mesures biométrologiques:

- spectrométrie gamma du thorax,

- excrétion fécale, complémentaire de celle-ci,

- excrétion urinaire et prélèvements nasaux, utiles en certaines circonstances,

- mais aussi les résultats d'examens portant sur le radiocontaminant lui-même et l'ambiance de travail.

A cet effet, ont été mises en place dans certains ateliers des opérations pilotes consistant en:

- mesures de granulométrie à l'inpacteur Andersen, complétées par l'examen au microscope électronique,

- mesure de la fraction inhalable par appareil de prélèvement individuel portatif,

- spectre de diffraction aux rayons $\mathrm{X}$, mesures de densité et de surface spécifique.

L'ensemble de ces résultats, encore trop parcellaires pour conclure, paraît montrer une assez bonne concordance avec le modèle pulmonaire avec, cependant, une excrétion urinaire souvent plus importante et une période biologique plus courte.

II est intéressant de constater que ces écarts vont dans le bon sens, c'est-à-dire celui d'une plus grande sécurité pour le personnel exposé. 


\section{BIBLIOGRAPHIE}

[1] ANSOBORLO E., BERARD Ph., CHALABREYSSE J. - Etude de l'exposition industrielle à des composés de l'uranium de classe $Y$ : $\cup_{2}\left(x \% U_{3} O_{8}\right)$. Versailles, 30 mai - 2 juin 1988. Radiat. Prot. Dos., 1989, 26 (1/4) 101-105.

[2] GROUPE DE TRAVAIL URANIUM (GT 8/1). - Valeurs de référence et leur application pour la surveillance de l'exposition professionnelle aux composés de l'uranium naturel. Rapport CEA-R-5370, 1986.

[3] INTERNATIONAL COMMISSION ON RADIOLOGICAL PROTECTION (ICRP) Limits for intakes of radionulides by workers (ICRP Publication 30, PT 1). Oxford: Pergamon Press, 1979.

[4] JOHNSON J.R., DUNFORD W. et al. - Canadian uranium fuel fabrication study. In: The assessment of radioactive contamination in man, Paris, 1984. Vienne: AlEA, $1985,297-323$.

[5] NENOT J.C., BEAU P.G. - Radiation protection in occupational health. (Safety Series $n^{\circ}$ 83). Vienne: AIEA, 1987.

[6] PIECHOWSKI J., MENOUX B. - Rétention et excrétion des radionucléides après incorporation par inhalation chez l'homme adulte. Rapport CEA-R-5266, 1984.

[7] PIECHOWSKI J., MENOUX B. - Protocole de surveillance individuelle pour les contaminations par inhalation de radionucléides en milieu professionnel. Rapport CEA-R-5305, 1984.

[8] SCHIEFERDECKER H., DILGER H., DOERFEL $H$. - Inhalation of $U$ aerosols from $\mathrm{UO}_{2}$ fuel element fabrication. Health Phys., 1985, 48 (1) 29-48. 